\title{
BMJ Open Comparative CT with stress manoeuvres for diagnosing distal isolated tibiofibular syndesmotic injury in acute ankle sprain: a protocol for an accuracy- test prospective study
}

To cite: Rodrigues JC, Santos ALG, Prado MP, et al. Comparative CT with stress manoeuvres for diagnosing distal isolated tibiofibular syndesmotic injury in acute ankle sprain: a protocol for an accuracy- test prospective study. BMJ Open 2020;10:e037239. doi:10.1136/ bmjopen-2020-037239

- Prepublication history for this paper is available online. To view these files, please visit the journal online (http://dx.doi. org/10.1136/bmjopen-2020037239).

Received 24 January 2020

Revised 02 May 2020

Accepted 21 July 2020
Check for updates

(C) Author(s) (or their employer(s)) 2020. Re-use permitted under CC BY-NC. No commercial re-use. See rights and permissions. Published by BMJ.

For numbered affiliations see end of article.

Correspondence to Dr João Carlos Rodrigues; joao.rodrigues@einstein.br

\section{ABSTRACT}

Introduction Although several imaging options are available for diagnosing syndesmotic injury, a fundamental question that guides treatment remains unanswered. Syndesmotic instability is still challenging to diagnose correctly, and syndesmotic disruption and true syndesmotic instability should be differentiated. Currently, imaging tests quickly diagnose severe syndesmotic instability but have difficulty in diagnosing mild and moderate cases. This study aims to investigate which strategy among an existing CT index test and two new add-on CT index tests with stress manoeuvres more accurately diagnoses syndesmotic instability. The secondary objective is to investigate the participants' disability outcomes by applying the Foot and Ankle Ability Measure questionnaire.

Methods and analyses This study of a diagnostic accuracy test will consecutively select individuals older than 18 years with a clinical diagnosis of a suspected acute syndesmotic injury. Three strategies of the CT index test (one in the neutral position and two with stress) will examine the accuracy using MRI as the reference standard. The external rotation and dorsiflexion of the ankle will guide the stress manoeuvres. A comparison of measurements between the injured syndesmosis and the uninjured contralateral side of the same individual will investigate the syndesmotic instability, by evaluating the rotational and translational relationships between the fibula and tibia. Sensitivity, specificity, area under the receiver operating characteristic curve and likelihood analyses will compare the diagnostic accuracies of the strategies.

Ethics and dissemination The Internal Review Board and the Einstein Ethics Committee approved this study (registered number 62100016.5.0000.0071). All participants will receive an oral description of the study's aim, and the choice to participate will be free and voluntary. Participants will be enrolled after they sign the written informed consent form, including the terms of confidentiality. The results will be presented at national
Strengths and limitations of this study

This study is the first to examine the accuracy and feasibility of CT with stress manoeuvres for diagnosing syndesmotic instability.

- The disability outcomes will be used to evaluate syndesmotic instability diagnosed by CT with stress manoeuvres as a prognostic factor.

- The limitations of this study include the use of MRI as the reference standard test, which, although not perfect, is estimated to have high accuracy compared with the gold-standard arthroscopy.

- The use of a clinical MRI protocol, which is partially optimised for syndesmosis, is another source of limitation.

- An inherent degree of imprecision and variability may occur when the participants themselves control dorsiflexion.

and international conferences and published in peerreviewed journals and social media.

Trial registration number ClinicalTrials.gov Registry (NCT04095598; preresults).

\section{INTRODUCTION}

One specific type of sprain is high ankle sprain with ligament damage to the distal tibiofibular syndesmosis. Syndesmotic lesions are the leading cause of persistent disability or chronic pain and frequently require longer recovery times and significantly more treatments than low lateral ankle sprains. ${ }^{12}$

The degree of instability in syndesmotic disruption guides the decision to operate or treat conservatively. ${ }^{3}$ Clinical examinations, routine radiographs, stress radiographs, CT in the neutral position (CTNP), weight-bearing 
computed tomography (WB-CT) and MRI are the diagnostic tools available for determining the correct treatment. The clinical diagnosis has limited evidence, and the best current practice considers that few clinical tests have any validity in identifying syndesmotic disruption. ${ }^{4}$ The mortise or anteroposterior radiographic view may promptly diagnose severe syndesmotic instability (SI) by identifying the widening of the articular space on the injured side relative to the contralateral unaffected side. However, SI may be underdiagnosed when routine radiography shows a typical articular relationship, and this method cannot reliably predict syndesmotic injuries. ${ }^{5}$ Stress radiographs are inaccurate for evaluating these injuries in a cadaveric models. ${ }^{6}$ Recently, WB-CT has emerged as a new modality for syndesmosis examination; however, WB-CT was not superior to CTNP in one study, ${ }^{7}$ and axial loading does not improve the diagnosis of instability. ${ }^{8}$ Although MRI can visualise and diagnose syndesmotic injury with high accuracy, ${ }^{9}$ MRI is an expensive examination that is not widely available. CTNP is more sensitive than radiography for detecting syndesmotic widening. ${ }^{10}$ Nevertheless, the traditional measurement of the anterior tibiofibular distance, obtained using CTNP, has an unsatisfactory area under the curve (AUC) performance of 0.56 for diagnosing SI. ${ }^{11}$

Despite the availability of these methods, SI is still challenging to diagnose correctly and syndesmotic ligament disruption and real SI should be differentiated. Comparative ankle CT with stress manoeuvres (CTSM) is an alternative approach that might improve SI diagnosis. To the best of our knowledge, the application of CTSM has not been previously described.

\section{Objectives and hypothesis}

The main aim is to investigate which strategy among an existing CTNP index test and two new add-on CTSM index tests (CTSM with extended knees (CTSM-EK) or flexed knees (CTSM-FK)) more accurately diagnoses SI. We hypothesised that the add-on CTSM-EK or CTSM-FK would be more accurate in diagnosing SI than CTNP alone.

The secondary objective is to investigate participants' disability outcomes by applying the Foot and Ankle Ability Measure (FAAM) questionnaire. We hypothesised that participants with SI, diagnosed using CTSM, will have worse disability outcomes than participants without SI.

\section{METHODS}

This study follows the guidelines of Standards for Reporting of Diagnostic Accuracy Studies. ${ }^{12}$

\section{Study design}

Single-centre diagnostic accuracy-test prospective study.

\section{Study setting}

The department of radiology, in partnership with the department of orthopaedics, conducted this study at a tertiary hospital with the approval of the Internal Review Board and the Ethics Committee.

\section{Participants}

\section{Inclusion criteria}

- Adults older than 18 years.

- One episode of an ankle sprain.

- The sprain episode occurred up to 3 weeks prior to imaging.

- A positive orthopaedic evaluation for suspected unilateral syndesmotic injury, defined as the presence of at least one of the following symptoms: pain during palpation of the distal tibiofibular syndesmosis, pain during the squeeze test (manual compression of the tibia and fibula in the middle third of the leg), pain during the external rotation test and an inability to stand on the tip of the affected foot.

\section{Exclusion criteria}

- Bilateral ankle sprain.

- Previous ankle surgery.

- Ankle fractures and dislocations (except avulsion fractures in ligamentous insertions or fracture of the posterior malleolus related to syndesmotic injury).

- Congenital or acquired ankle deformities.

- Infection, inflammatory or neuropathic ankle arthropathies.

\section{Participant selection and recruitment}

A consecutive sample of individuals with suspected syndesmotic disruption visiting the foot and ankle outpatient clinic will be referred to the radiology department for a CT and an MRI. A research assistant (RA) will screen and interview all participants for eligibility and their willingness to participate, clarifying the research aims and inviting those individuals who meet the inclusion criteria to enrol. The RA will also describe all the study details, answer all questions about the objectives, risks, benefits and confidentiality and read aloud the informed consent for which the individuals who agree to participate will sign and date. The RA will attach a copy of the informed consent form to the medical record and handover a second copy to the participants. Participants will provide demographic data and will complete preexamination forms before undergoing imaging examinations. The reasons for exclusion or refusal to participate will be recorded. Figure 1 shows a flowchart delineating the study procedures.

\section{CT examination technical parameters}

An Aquilion ONE V.6 scanner (Toshiba Medical Systems, Tochigi, Japan) with 320 channels will be used to perform all the examinations with the following technical parameters: a volumetric acquisition, $120 \mathrm{kV}, 150 \mathrm{~mA}, 0.5 \mathrm{~s}$ rotation time, $0.5 \mathrm{~mm}$ slice thickness, $0.25 \mathrm{~mm}$ interpolation, 320-detector rows, medium or large field of view (FOV) and fine filter for bone. The lowest possible irradiation dose will produce images of diagnostic quality. The feet 


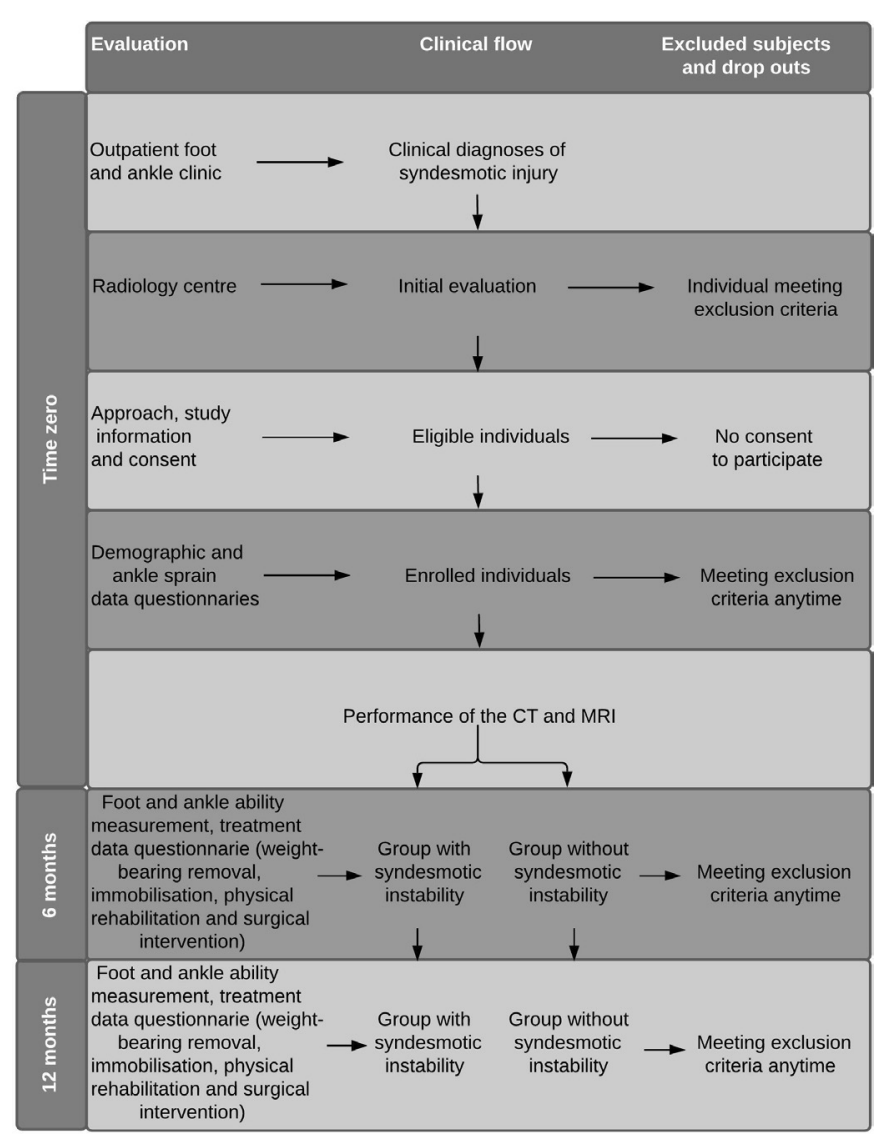

Figure 1 Clinical pathway for the syndesmosis study. CTSM, CT with stress manoeuvres.

of the participants will be simultaneously scanned in the same FOV.

\section{Existing index test (CTNP)}

In the neutral phase, the feet will be parallel and perpendicular to the long axis of the legs. The knees will be in an extended position.

\section{New index test (CTSM-EK)}

In the first stress phase, the researchers will control external rotation by placing the feet at 45 degrees using an angle metre and a vertical line as a reference. Voice commands will instruct the participant to maintain maximum dorsiflexion to the limit of tolerable pain during image acquisition. The knees will be in an extended position.

\section{New index test (CTSM-FK)}

In the second stress phase, the researchers will control external rotation by placing the feet at 45 degrees using an angle metre and a vertical line as a reference. Voice commands will instruct the participant to maintain maximum dorsiflexion to the limit of tolerable pain during image acquisition. A support pad will maintain the knees in a flexed position at 45 degrees.

\section{Acrylic board}

Investigators will perform the CTNP, CTSM-EK and CTSM-FK in a standardised manner. All participants will be scanned in the supine position with their feet supported on an acrylic board (Medintec, Mogi das Cruzes, Brazil) connected to a pair of side strings of adjustable length. The researchers will ask the participants to hold the manual support at the proximal ends of the strings and will give verbal commands through the room's speakers to pull strings and perform dorsiflexion at the appropriate times.

\section{Participants' training stress manoeuvres and feasibility assessment}

The technicians will guide participants to train dorsiflexion by simulating the movement of the feet, pulling strings just before image acquisition. Difficulties in performing the stress manoeuvres, including pain exacerbation, motion artefacts, image repetition, total examination duration and dropout, will be used to assess the feasibility of the new index test.

\section{CTNP, CTSM-EK and CTSM-FK reading parameters}

Measurements comprising six distances, two ratios and two angles will determine the anatomic tibiofibular relationship, as described by Nault et al. ${ }^{13}$ According to LaMothe et $a l,{ }^{14}$ posterior translation of the fibula is an important component of the SI. The evaluation described by Nault et $a l^{3}$ is capable of examining the rotational and multiplanar translations of the fibula, including the anteroposterior and lateral planes. Figure 2 shows the distances, ratios and angles. A reference line $1 \mathrm{~cm}$ above the tibial plafond will establish the correct plane for all the measurements, except for the second angle, which will occur in the plane of the tibial plafond. Another measurement will determine the smallest distance between the fibula and the tibia in the plane of the tibial plafond, as described by Ahn $e t a l .{ }^{11}$ All the measurements will occur in a standardised manner in the CTNP, CTSM-EK and CTSM-FK. Differences equal to or greater than $1 \mathrm{~mm}$ for distances and 2 degrees for angles between the injured and uninjured ankles of the same individual will define the cutoff values for a positive test. Differences less than $1 \mathrm{~mm}$ and 2 degrees will define the cut-off values for a negative test. Although there are no previous studies on CTSM, the researchers adopted cut-off points based on a recent study comparing injured and uninjured syndesmoses, which showed significant differences in the distances and angles between groups estimated at $1 \mathrm{~mm}$ and 2 degrees, respectively. ${ }^{15}$

\section{MRI examination technical parameters}

A phased-array dedicated coil on a 1.5-T magnet HDX (GE Healthcare, Milwaukee, USA) will be used to perform all of the examinations with the following sequences: sagittal T1-weighted (time of repetition/time of echo, 542/9; number of excitations, 1 ; matrix, $320 \times 256$; thickness, $4 \mathrm{~mm}$; FOV, $10 \mathrm{~cm}$ ), sagittal T2-weighted fat suppressed $(3000 / 39,2,384 \times 224,4,10)$; axial T2-weighted fat suppressed $(3483 / 48,2,384 \times 224,4$ and 10$)$. Two optimised sequences with a $3 \mathrm{~mm}$ slice thickness will also be 

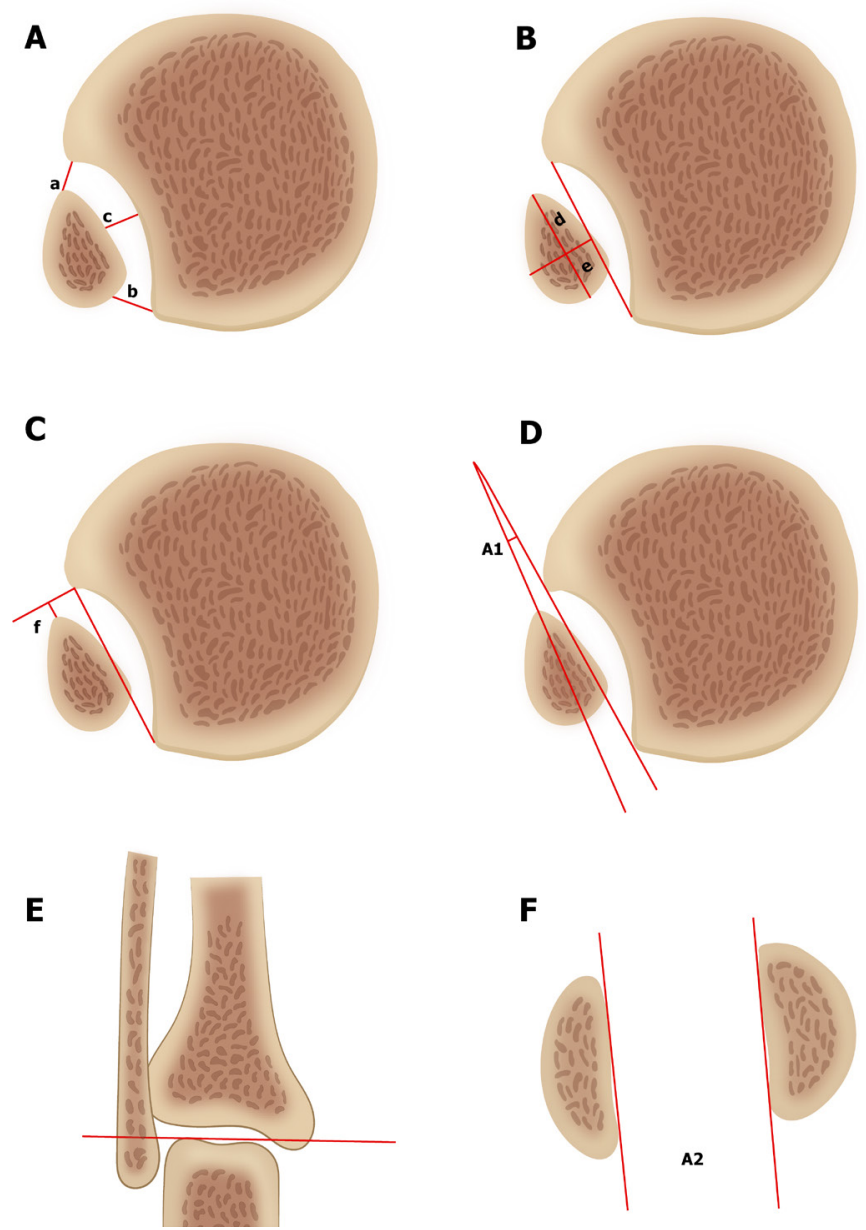

$\mathbf{F}$

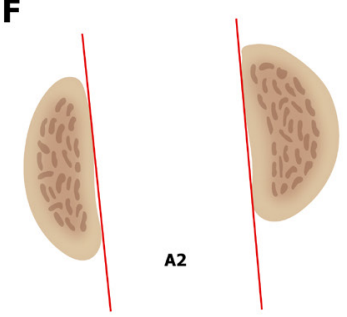

Figure 2 A, B, C and D images represent the axial views of a normal syndesmosis $1 \mathrm{~cm}$ proximal to the tibial plafond with six measures $(A-F)$ and one angle $(A 1)$. Image $(E)$ depicts the reference line on the tibial plafond and $(F)$ the corresponding second angle (A2) measured.

used: coronal T2-weighted fat-suppressed (3000/39, 2, $384 \times 224,3$ and 10$)$ and coronal oblique proton density weighted $(2840 / 35,2,384 \times 224,3$ and 10$)$.

\section{Reference test (MRI)}

The standard protocol will acquire all of the ankle MRIs for participants suspected to have a syndesmotic injury as described below. Participants will be scanned with their knees in extension and ankles in a neutral position. Two studies comparing the accuracy of MRI with arthroscopy have shown that MRI is highly sensitive and specific for evaluating syndesmotic injury. ${ }^{9} 16$ The arthroscopic examination facilitates a correct diagnosis and treatment and is considered the best reference standard for syndesmotic injuries. ${ }^{17}$ However, in this study, participant selection is based on the ankle sprain condition and physical examination, which has limited accuracy. ${ }^{4}$ A significant proportion of uninjured syndesmosis will be selected with the alternative diagnoses of a lateral collateral ligament injury. The application of arthroscopy to the alternative diagnosis group would have been difficult to justify ethically because the index and reference tests will presumably show negative results. Even a minimally invasive procedure, such as the arthroscopic examination, may have complications and is not risk free. ${ }^{18} 19$

\section{MRI reading parameters}

The investigators prespecified the result categories by classifying syndesmotic ligaments (anterior inferior tibiofibular, posterior inferior tibiofibular and interosseous), lateral collateral ligaments (anterior talofibular, calcaneofibular and posterior talofibular) and deltoid ligaments (superficial and deep layer) as grade 0 (normal ligament), grade I (soft tissue oedema around the ligament but still intact), grade II (partial tear with high signal intensity and thickening) or grade III (complete ligament tear with avulsion or discontinuity) injury. ${ }^{20}$

\section{FAAM questionnaire}

During the follow-up, the RA will contact participants 6 and 12 months after CT and will ask them to answer the FAAM questionnaire by phone or online. The FAAM questionnaire will quantify the extent of disability. ${ }^{21}$ The FAAM is a reliable, responsive and valid tool for evaluating the physical activity of individuals with a variety of disorders of the leg, foot and ankle. A systematic review of the literature identified the FAAM as one of the most appropriate patient-assessed tools to quantify functional ankle disabilities. ${ }^{22}$ The FAAM questionnaire consists of two subscales with 29 questions in total. The Activities of Daily Living (ADL) subscale comprises 21 questions and assesses an individual's ability to stand, squat or climb stairs. The Sports subscale comprises eight questions and assesses the ability to run, jump and land. The two subscales are reported separately, with a score ranging from 0 to 84 points for the ADL subscale and 0-32 points for the Sports subscale. The range of scores for each subscale changes from the lowest score $(0)$ to the highest (100) in percentage. Higher scores represent an improvement in the outcomes of both subscales. The exposure factor of interest will be SI, as detailed in the CTNP, CTSM-EK and CTSM-FK reading parameters. Data will be collected on the following covariates related to the participants' characteristics: age (years), gender (male or female) and body mass index (BMI). Data will also be collected on the covariates related to treatment: weight-bearing restrictions (partial or total), immobilisation (cast or robot-foot), physical rehabilitation (number of sessions) and surgical intervention (screws or suturebottom fixation). As these data may change over time, the analysis will include data recorded at the time of the last assessment. In this observational cohort, researchers will not control for all of these covariates but will adequately monitor and analyse their effects on the disability outcomes in both groups with or without the exposure factor.

\section{Blinding and observation bias}

Five groups of individuals will be involved in this study: participants, orthopaedists, radiologists, RA, index and 
reference standard technicians and a statistician. The following actions will be taken to avoid observation bias. The orthopaedists will perform the physical examination while blinded to the index or reference standard test results. The radiologists will read the index tests while blinded to the reference standard test results, participant identification, physical examination results and laterality of the ankle with the suspected syndesmotic lesion. The radiologists will read the reference standard tests while blinded to the participant identification, physical examination results and index test results. The RA will collect the questionnaires while blinded to the index or reference standard test results and the physical examination results. The technician will perform the index tests while blinded to the reference standard test or physical examination results. Another technician will perform the reference standard test while blinded to the index test or physical examination results. Labelling the variables with non-identifying terms (such as A and B) will blind the statistician to the results. Researchers will not communicate the group assignment to the participants.

\section{Patient and public involvement}

Neither the participants nor the public were involved in planning or developing this protocol. Participants will receive information about the knowledge obtained in this study through correspondence or emails. Open lectures in the hospital auditorium and social media communication will disseminate the results to the public.

\section{Statistical analysis}

\section{Descriptive analyses}

Absolute frequencies and percentages will describe categorical variables. Means, SD, medians, quartiles and minimum and maximum values will define numerical variables depending on the data distribution.

\section{Inference analyses of diagnostic accuracy}

Sensitivity, specificity, AUC and likelihood ratios (LRs) will be calculated to compare the diagnostic accuracy of the three strategies. The first strategy will test the diagnostic accuracy of the existing index CTNP alone, the second will examine the add-on index CTSM-EK after CTNP and the third will assess the add-on index CTSM-FK after CTNP. For statistical analyses, the 'either positive' rule will be adopted, whereby the add-on test is positive if either component test is positive. The relative and absolute differences in sensitivity and specificity will compare the performance of the three strategies considering the paired nature of the data. The add-on strategies will exhibit superior accuracy if the positive LRs are higher than the existing strategy and the CI for the LR does not contain 1 . The existing strategy will exhibit superior accuracy if the negative LR is less than the add-on strategies and the CI for the LR does not include 1.

Inconclusive and missing index test data

Inconclusive invalid results of the index tests may occur and will be regarded as uninterpretable when the critical characteristic of the test is corrupted or has questionable validity as a result of a low-quality procedure. A missing index result may occur when a participant is included in the study but does not complete the index test. Missing index results will be treated in the same manner as inconclusive invalid results, and both will be reported separately from the valid results. The underlying causes will be reviewed and discussed by considering the participant's disease status, an alternative target condition or whether they are unrelated to the participant. A test is valid (technically appropriate) but inconclusive when the result is neither positive nor negative. For the continuous index test measurements, 'rule in' and 'rule out' will define thresholds after considering an interval of valid, inconclusive test values. The cross-table will consider the number of participants with positive and negative results for the disease in each category, accommodating new groups in extra rows. Paired histograms, dot plots or cumulative distribution graphs will show the distribution of the test results stratified by disease status. Multilevel or stratum-specific LRs will summarise the performance of the index tests on a quantitative test scale to analyse the valid inconclusive results, thus reducing the tendency of spectrum bias.

\section{Missing and inconclusive reference standard test data}

In this study, a small, incidental, random amount of missing and inconclusive, invalid reference standard test data unrelated to the participants' characteristics or index test results may occur. The percentage of missing or invalid data will be reported separately from the valid results, including the distribution of participants' characteristics and index test results in individuals with or without a reference standard test. A sensitivity analysis will measure the effect of these missing or invalid data. After confirming this scenario, the researchers will perform a complete case analysis by including participants with index and reference standard tests and excluding participants with missing or invalid reference standard tests. If a complete case analysis yields biased accuracy results, an analytical approach will use the available data to reconstruct the missing or invalid data through inverse probability weighting or multiple imputations.

\section{Analysis of variability}

A subgroup analysis of participants will assess the sources of variability in the accuracy of the index test. The degree of the severity of the ankle sprain determined in the reference standard test will prespecify three subgroups based on the number of syndesmotic ligaments damaged. Isolated lesions of the anterior tibiofibular ligament will be defined as a mild sprain. Injuries involving the anterior tibiofibular and interosseous ligaments will define a moderate sprain. Lesions involving the anterior tibiofibular, interosseous and posterior tibiofibular ligaments will indicate a severe sprain. The index test performance is expected to be more accurate for patients with the higher degrees of sprain than for patients with lower 
degrees of sprain. Participant-controlled dorsiflexion during the stress manoeuvre may be another source of variability. The current acrylic board system does not allow researchers to control dorsiflexion during the examination, but they will register this measure in the acquired images and verify the effect on the accuracy of the results. Pain aggravation reported by the participant will prespecify one subgroup and pain non-aggravation the other subgroup. The pain aggravation subgroup may experience difficulty performing dorsiflexion and lower accuracy results compared with participants in the pain non-aggravation subgroup may be observed. Sensitivity, specificity, AUC and LRs will be used to examine the diagnostic accuracy of the subgroups.

\section{Interrater analysis}

Two observers will independently read the index tests; two other observers will independently read the reference tests. The intraclass correlation coefficient will verify the agreement between observers regarding the data extracted from the index test, and the Kappa coefficient will confirm the agreement between observers related to data derived from the reference standard test. A second consensus reading will be performed to solve discordant cases.

\section{Inference analyses for the FAAM questionnaire}

Mixed linear models or generalised linear models in multiple approaches will evaluate the association of the SI findings, participants' characteristics (age, gender and BMI) and treatment (weight-bearing restriction, immobilisation, physical rehabilitation and surgery) with the change in the extent of disability (FAAM) at 6 and 12 months after CT. These models are appropriate for measurements obtained from the same participant at different times. The effect estimates will present the results (change in mean functional disability) with $95 \%$ CIs. A two-sided t-test will compare the changes in the FAAM score between the groups.

\section{Sample size calculation}

The sample size calculation will consider the comparison of the primary objective (diagnostic accuracy) and the secondary objective (change in the FAAM disability score). The largest sample needed to meet the primary or secondary objectives will define the study sample size.

A previous study reported an AUC performance of 0.56 relative to the CTNP. ${ }^{11}$ Assuming the null hypothesis that the existing CTNP test has an AUC of 0.56, this study proposes the alternative hypothesis that a new CTSM test will display superior accuracy, which is estimated as an AUC of 0.80 . The full sample size required to detect a difference between these two outcomes is estimated as 39 , with a $1: 2$ allotment ratio between the groups (13 and 26 participants per group, respectively).

A previous study reported an effect size of the change score paired data for the FAAM Sports subscale of 15, with a SD of the change of 28 in one group and 23 in the other. ${ }^{23}$ Using a two-sample paired t-test to compare the changes in the Sports subscale, the required full sample size is 111 , with a
1:2 allocation ratio between the groups ( 37 and 74 cases per group, respectively). Based on the same study ${ }^{23}$ and applying the same methodology, the required full sample size for the ADL subscale is 60 participants.

The sample of 111 individuals required for the Sports subscale defined the study sample size after contemplating the sample calculations of the ADL subscale and the AUC. Accounting for a possible 20\% loss during follow-up, the investigators intend to include 133 participants in the study.

Once the study has reached a follow-up of 67 participants, the sample size needed to compare the groups will be recalculated based on both the SD and the proportion of syndesmotic lesions observed.

\section{Software and thresholds}

The MedCalc Statical Software, version 19.4.0 (MedCalc Software Ltd, Ostend, Belgium), will perform the analyses, with $80 \%$ power to test group differences at a significance level of $5 \%$ and $95 \%$ CIs.

\section{ETHICS AND DISSEMINATION}

The Internal Review Board approved this study, which will be conducted according to the requirements of the Research Ethics Committee (62100016.5.0000.0071) and the recommendations established by the Declaration of Helsinki (2013). Participation is free and voluntary, and all recruited individuals will receive a verbal explanation of the purpose of the study. This study will enrol participants after they have signed the informed consent form, including confidentiality terms. Researchers will present the results at national and international conferences and will publish the results in peerreviewed scientific journals and social media to disseminate knowledge.

\section{DISCUSSION}

Although several imaging options are available for syndesmotic injury diagnosis, a fundamental question guiding treatment remains unanswered. Currently, imaging tests readily diagnose severe SI but have difficulty diagnosing mild and moderate cases. Presumably, the correct treatment is not being applied to a significant proportion of individuals. Undiagnosed and untreated mild and moderate SI are considered the primary sources of unfavourable outcomes. If this study confirms CTSM as an accurate method for diagnosing SI, a new approach to investigate challenging cases may become available, and more individuals may benefit from correct treatment, reducing the burden of unfavourable outcomes. An algorithm combining clinical suspicion, MRI findings and CTSM protocol may be the correct and precise method to diagnose SI.

\section{Strengths}

To the best of our knowledge, this study is the first to test the accuracy of CTSM examination in diagnosing SI and the feasibility of stress manoeuvres, which will be evaluated based on 
the pain perception reported by participants in addition to the objective parameters measured by the performing technician. This study is also the first to test SI, diagnosed with CTSM, as a prognostic factor for disability outcomes. This assessment is not confined exclusively to exposure (with or without SI). Participants' demographics and treatment characteristics will also be investigated to determine their effects on the outcomes.

\section{Limitations}

This study uses MRI as the reference standard test, which, although not perfect, has a high estimated accuracy compared with the gold-standard arthroscopy. ${ }^{16}$ The use of a clinical MRI protocol, partially optimised for syndesmosis, is another limitation.

An inherent degree of imprecision and variability may occur when the participants themselves control dorsiflexion.

The lack of follow-up imaging to document the progression of instability, provide long-term outcomes and predict the strength of the initial imaging data is another limitation.

A loss-to-follow-up bias may occur because the unexposed group is expected to experience more pronounced symptom relief, which may prevent individuals from participating throughout the follow-up period.

\section{Author affiliations}

${ }^{1}$ Radiologia, Hospital Israelita Albert Einstein, Sao Paulo, Brazil

${ }^{2}$ Radiologia, Universidade de São Paulo Hospital das Clínicas, Sao Paulo, Brazil

${ }^{3}$ Programa Locomotor, Hospital Israelita Albert Einstein, Sao Paulo, Brazil

${ }^{4}$ Ortopedia, Universidade de São Paulo Hospital das Clínicas, Sao Paulo, Brazil

\section{Correction notice ORCiDs for the authors are added.}

Acknowledgements The authors gratefully acknowledge the contribution of Gilberto Szarf for providing all the necessary support to turn an idea into a real project; Leonardo Kamibeppu and Fabio Augusto for all their assistance with biomedical activities; Elivane da Silva Victor and Ana Carolina Cintra Nunes Mafra for their valuable contributions to the statistical plane; Rosemeire Pereira Bezerra and Giovanna de Souza Mendes for all their assistance with administrative issues and organisation of the questionnaires; Maria Angela M. Barreiros for preparing the flowchart, and American Journal Experts for editing this paper for the proper English language, grammar, punctuation, spelling and overall style.

Contributors JCR conceived the study, participated in its conception, design and coordination and drafted the manuscript. ALGS and MPP participated in the conception and design of the project and writing the paper. RAM, JFMA, DdCSB and LAR participated in the design and coordination of the study and contributed to the critical revision of the article. AdAeC, MKD, ML and MF participated in the design and critical review of the manuscript. All authors read and approved the final version of the paper.

Funding The authors have not declared a specific grant for this research from any funding agency in the public, commercial or not-for-profit sectors.

\section{Competing interests None declared.}

Patient and public involvement Patients and/or the public were not involved in the design, or conduct, or reporting, or dissemination plans of this research.

Patient consent for publication Not required.

Provenance and peer review Not commissioned; externally peer reviewed.

Open access This is an open access article distributed in accordance with the Creative Commons Attribution Non Commercial (CC BY-NC 4.0) license, which permits others to distribute, remix, adapt, build upon this work non-commercially, and license their derivative works on different terms, provided the original work is properly cited, appropriate credit is given, any changes made indicated, and the use is non-commercial. See: http://creativecommons.org/licenses/by-nc/4.0/.

\section{ORCID iDs}

João Carlos Rodrigues http://orcid.org/0000-0002-7107-2621

Alexandre Leme Godoy Santos http://orcid.org/0000-0002-6672-1869

Marcelo Pires Prado http://orcid.org/0000-0003-0325-8050

José Felipe Marion Alloza http://orcid.org/0000-0002-7664-2064

Laercio Alberto Rosemberg http://orcid.org/0000-0003-4395-1159

Durval do Carmo Santos Barros http://orcid.org/0000-0002-5210-3605

Adham do Amaral e Castro http://orcid.org/0000-0003-0649-3662

Marco Kawamura Demange http://orcid.org/0000-0003-1999-9478

Mario Lenza http://orcid.org/0000-0003-3497-2206

Mario Ferretti http://orcid.org/0000-0002-5298-2486

\section{REFERENCES}

1 Gerber JP, Williams GN, Scoville CR, et al. Persistent disability associated with ankle sprains: a prospective examination of an athletic population. Foot Ankle Int 1998;19:653-60.

2 Boytim MJ, Fischer DA, Neumann L. Syndesmotic ankle sprains. Am $J$ Sports Med 1991;19:294-8.

3 van Dijk CN, Longo UG, Loppini M, et al. Conservative and surgical management of acute isolated syndesmotic injuries: ESSKA-AFAS consensus and guidelines. Knee Surg Sports Traumatol Arthrosc 2016;24:1217-27.

4 van Dijk CN, Longo UG, Loppini M, Maltese L, Denaro V, Ciuffreda $\mathrm{M}$, et al. Classification and diagnosis of acute isolated syndesmotic injuries: ESSKA-AFAS consensus and guidelines. Knee Surg Sports Traumatol Arthrosc 2016;24:1200-16.

5 Krähenbühl N, Weinberg MW, Davidson NP, et al. Imaging in syndesmotic injury: a systematic literature review. Skeletal Radiol 2018;47:631-48.

6 Beumer A, Valstar ER, Garling EH, et al. External rotation stress imaging in syndesmotic injuries of the ankle: comparison of lateral radiography and radiostereometry in a cadaveric model. Acta Orthop Scand 2003;74:201-5.

7 Hamard M, Neroladaki A, Bagetakos I, et al. Accuracy of conebeam computed tomography for syndesmosis injury diagnosis compared to conventional computed tomography. Foot Ankle Surg 2020;26:265-272.

8 Krähenbühl N, Bailey TL, Weinberg MW, et al. Is load application necessary when using computed tomography scans to diagnose syndesmotic injuries? A cadaver study. Foot Ankle Surg 2020;26:3-4.

9 Oae K, Takao M, Naito K, et al. Injury of the tibiofibular syndesmosis: value of MR imaging for diagnosis. Radiology 2003;227:155-61.

10 Ebraheim NA, Lu J, Yang H, et al. Radiographic and CT evaluation of tibiofibular syndesmotic diastasis: a cadaver study. Foot Ankle Int 1997;18:693-8.

11 Ahn T-K, Choi S-M, Kim J-Y, et al. Isolated syndesmosis diastasis: computed tomography scan assessment with arthroscopic correlation. Arthroscopy 2017;33:828-34.

12 Cohen JF, Korevaar DA, Altman DG, Gatsonis CA, Levine D, et al. STARD 2015 guidelines for reporting diagnostic accuracy studies: explanation and elaboration. BMJ Open 2016;6:e012799.

13 Nault M-L, Hébert-Davies J, Laflamme G-Y, et al. CT scan assessment of the syndesmosis: a new reproducible method. $J$ Orthop Trauma 2013;27:638-41.

14 LaMothe JM, Baxter JR, Karnovsky SC, et al. Syndesmotic injury assessment with lateral imaging during stress testing in a cadaveric model. Foot Ankle Int 2018;39:479-84.

15 Nault M-L, Gascon L, Hébert-Davies J, et al. Modification of distal tibiofibular relationship after a mild Syndesmotic injury. Foot Ankle Spec 2017;10:133-8.

16 Takao M, Ochi M, Oae K, et al. Diagnosis of a tear of the tibiofibular syndesmosis. The role of arthroscopy of the ankle. J Bone Joint Surg $\mathrm{Br}$ 2003;85:324-9.

17 Lubberts B, Guss D, Vopat BG, et al. The arthroscopic syndesmotic assessment tool can differentiate between stable and unstable ankle syndesmoses. Knee Surg Sports Traumatol Arthrosc 2020;28:193-201.

18 Ferkel RD, Small HN, Gittins JE. Complications in foot and ankle arthroscopy. Clin Orthop Relat Res 2001:89-104.

19 Carlson MJ, Ferkel RD. Complications in ankle and foot arthroscopy. Sports Med Arthrosc Rev 2013;21:135-9.

20 Sharif B, Welck M, Saifuddin A. MRI of the distal tibiofibular joint. Skeletal Radiol 2020;49:1-17.

21 Moreira TS, Magalhães LdeC, Silva RD, et al. Translation, crosscultural adaptation and validity of the Brazilian version of the foot and ankle ability measure questionnaire. Disabil Rehabil 2016;38:2479-90. 
22 Eechaute C, Vaes P, Van Aerschot L, et al. The clinimetric qualities of patient-assessed instruments for measuring chronic ankle instability: a systematic review. BMC Musculoskelet Disord 2007;8:6.
23 Martin RL, Irrgang JJ, Burdett RG, et al. Evidence of validity for the foot and ankle ability measure (FAAM). Foot Ankle Int 2005;26:968-83. 